\title{
Pseudoelastic mesh-moving using a general scenario of the selective mesh stiffening
}

\author{
Daisuke Ishihara $^{1 *}$, Atsushi Goto ${ }^{1}$, Minato Onishi ${ }^{1}$, Tomoya Niho ${ }^{1}$, Tomoyoshi Horie ${ }^{1}$ \\ ${ }^{1}$ Department of Mechanical Information Science and Technology, Kyushu Institute of Technology \\ *ishihara@mse.kyutech.ac.jp
}

Received: October 17, 2018; Accepted: January 17, 2019; Published: March 15, 2019

\begin{abstract}
The selective mesh stiffening in this study changes the stiffness of the element based on both the element area and shape. It includes the stiffening in the previous studies as a specific case, and leads to a general scenario in the pseudoelastic mesh-moving. This scenario gives better mesh quality in the mesh-moving of a rectangular domain with a structure consisting of a square and a fin undergoes large rotations. This is because the shear deformation of the element is adaptively considered.
\end{abstract}

Keywords: Finite element method, Pseudoelastic mesh-moving, Interface tracking

\section{Introduction}

Interface-tracking methods are suitable for such FSI problems, for which moving interfaces should be described accurately. The mesh must be updated and deform to follow the interface motion. In the pseudoelastic mesh-moving, the fluid mesh is modeled as a pseudoelastic mesh, and finite element linear elastic analysis is performed using the displacement on the moving boundary as the elementary boundary condition. The resulting nodal displacement is used to move the corresponding node. In the pseudoelastic mesh-moving, the selective mesh stiffening is used to maintain the mesh quality.

In this paper, a general scenario of the selective mesh stiffening is proposed. In this scenario, the stiffness of the element increases based on both the area and aspect ratio of the element. This stiffening includes the Jacobian-based stiffening [1] and the minimum-heightbased stiffening [2] as a specific case. The superior ability of the proposed scenario to maintain the mesh quality is demonstrated using a rectangular domain containing a structure consisting of a square and a fin that undergoes large deformation $[3,4]$. 


\section{Selective mesh stiffening in the pseudoelastic mesh-moving}

Let us consider $\Omega$ as the spatial domain and $\Gamma$ as its boundary. In the pseudoelastic mesh-moving, a pseudoelastic mesh occupies $\Omega$, and its displacement along the moving boundary is imposed on $\Gamma$ as the essential boundary condition. The equilibrium equation for the elastic body is considered to be the governing equation for the pseudoelastic material. Hooke's law is used to describe the relationship between the stress and strain tensors. Applying the finite element formulation to the governing equation, the discrete equation system can be obtained in matrix form. No external forcing function is defined to selectively handle mesh motion.

Using a linearly interpolated triangular element, the stiffness matrix $\mathbf{K}$ is given as

$$
\mathbf{K}=\sum_{e} \kappa_{e} \mathbf{K}_{e},
$$

where $\kappa_{e}$ is the stiffening coefficient for each element, $\mathbf{K}_{e}$ is the elemental stiffness matrix. $\kappa_{e}$ is used to control the stiffness of each element. In the Jacobian-based stiffening [1] and the minimum-height-based stiffening [2], $\kappa_{e}$ is given, respectively, as

$$
\kappa_{e}=\left(\frac{A_{0}}{A_{e}}\right)^{\chi},
$$

and

$$
\kappa_{e}=\left(\frac{h_{0}}{h_{e}^{\min }}\right)^{\chi},
$$

where $\chi$ is the nonnegative stiffening power, $A_{e}$ is the area of element $e, h_{e}^{\text {min }}$ is the minimum height for element $e, A_{0}$ is an arbitrary scaling parameter with the dimension of area, and $h_{0}$ is an arbitrary scaling parameter with the dimension of length.

\section{General scenario of the mesh stiffening}

The mesh quality can be evaluated using the following measures [1]:

$$
\max f_{e}^{A},
$$

and

$$
\max f_{e}^{R},
$$

which are the changes in the area and shape of the element $e$, respectively, as 


$$
f_{e}^{A}=\left|\log \left(\frac{A_{e}}{A_{e}^{o}}\right)\right|
$$

and

$$
f_{e}^{R}=\left|\log \left(\frac{R_{e}}{R_{e}^{o}}\right)\right|
$$

where $A_{e}$ and $R_{e}$ are the area and aspect ratio of element $e$, respectively, and the superscript $o$ referes to the undeformed mesh. $R_{e}$ is defined as $\left(l_{\mathrm{e}}{ }^{\max }\right)^{2} / A_{e}$, where $l_{\mathrm{e}}{ }^{\max }$ is the maximum edge for element $e$. The fundamental parameters in these mesh quality measures are $A_{e}$ and $R_{e}$. Therefore, these two parameters can be used to maintain the mesh quality in $\kappa_{e}$ as

$$
\kappa_{e}=\left(\left(\frac{A_{0}}{A_{e}}\right)^{\chi_{1}} R_{e}^{\chi_{2}}\right)^{\chi_{3}},
$$

where $\chi_{1}$ and $\chi_{2}$ satisfy the relationship $\chi_{1}+\chi_{2}=1$, since they define the weights of the element quality parameters $A_{e}$ and $R_{e}$. (5) reduces to (2a) or the Jacobian-based stiffening coefficient for $\chi_{1}=1.0$ and $\chi_{2}=0.0$ as

$$
\kappa_{e}=\left(\frac{A_{0}}{A_{e}}\right)^{\chi_{3}}
$$

and reduces to $(2 \mathrm{~b})$ or the minimum-height-based stiffening coefficient for $\chi_{1}=0.5$ and $\chi_{2}=$ 0.5 as

$$
\kappa_{e}=\left(\left(\frac{A_{0}}{A_{e}}\right)^{0.5} R_{e}^{0.5}\right)^{\chi_{3}}=\left(\frac{A_{0}^{0.5} l_{e}^{\max }}{A_{e}}\right)^{\chi_{3}}=\left(\frac{2 A_{0}^{0.5}}{h_{e}^{\min }}\right)^{\chi_{3}},
$$

where $R_{e}=\left(l_{\mathrm{e}}^{\max }\right)^{2} / A_{e}$ is used to obtain the third expression, and $A_{e}=h_{\mathrm{e}}{ }^{\min } l_{\mathrm{e}}{ }^{\max } / 2$ is used to obtaion the last expression. Therefore, The proposed stiffening coefficient (5) gives the quite general scenario.

Figure 1 shows the examples of the deformation modes of the element and the reactions of the properties in $\kappa_{e}$ to them. As shown in this figure, the combination of the area and the aspect ratio can react to all the modes. Therefore, it is expected that the proposed $\kappa_{e}$ will show the superior ability, especially, in the case of the large shear deformation, since only the aspect ratio can react to the shear deformation. 
A: Elongation / Contraction (Incompressible)
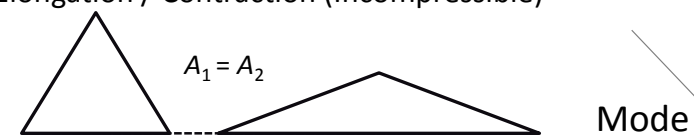

Area Minimum Aspect

B: Elongation / Contraction (Poisson's Ratio $=0$ )

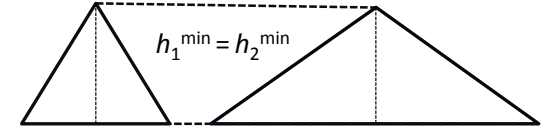

A $\times \quad O \quad O$

C: Expansion / Compression (Geometric Similarity)

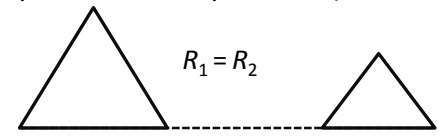

D: Shear
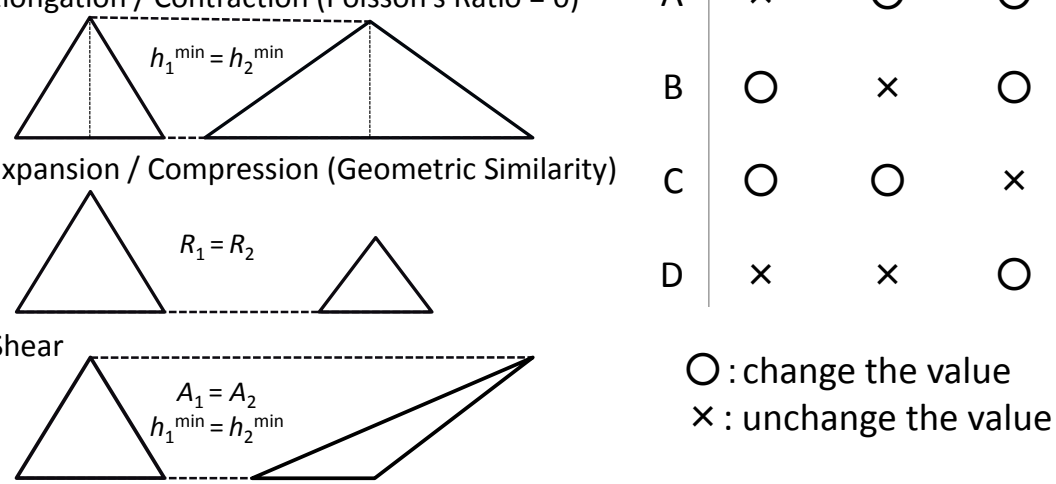

$O$ : change the value

$x$ : unchange the value

Figure 1: Examples of the deformation modes of the element and the reactions of the properties in $\kappa_{e}$ to them.

\section{Numerical examples}

\subsection{Two-dimensional rotation}

A rectangular domain including a rigid body consisting of a square and a fin was considered, as shown in Fig. 2. This domain was descritized using linearly interpolated triangular elements with the number of nodes and elements 4641 and 8 996, respectively. A rotational displacement $\theta_{z}$ about the $z$-axis was applied to the rigid body incrementally. $\chi_{3}$ for each $\left(\chi_{1}, \chi_{2}\right)$ values was obtained as the value that minimizes the mesh quality measure of (3).

Figures 3 and 4 shows the changes of the mesh quality measures plotted against the rotation $\theta_{z}$. As shown in these figures, the stiffening coefficient with $\left(\chi_{1}, \chi_{2}\right)=(0.3,0.7)$ showed the best performance to maintain the element area and shape under large rotations. From this observation, the proposed scenario with $\chi_{1}<\chi_{2}$ shows better performance than the previous stiffening in terms of maintaining the mesh quality under large rotations. Figures 5 and 6 show the moved meshes near the structure in the case of the maximum rotation $\theta_{z}=150^{\circ}$, where the equivalent shear strain fields are drawn. As shown in these figures, the mesh was twisted around the structure after the rotation. This type of motion induced a large shear deformation in the vicinity of the structure. The Jacobian-based stiffening generated the larger shear deformation as shown in Fig. 5. This is because the shear deformation does not contribute to the area change, and the Jacobian-based stiffening uses only the evaluation for the area as discussed in Section 3. Similary, the minimum-height-based stiffening uses only the equally weighted evaluation for the area and the aspect ratio irrespective of the change of the magnitude of the shear deformation. On the contrary, the proposed scenario can change the weighted evaluation for the area and the aspect ratio of each element according to the magnitude of the shear deformation. Therefore, the proposed scenario reduces the shear deformation as shown in Fig. 6. 


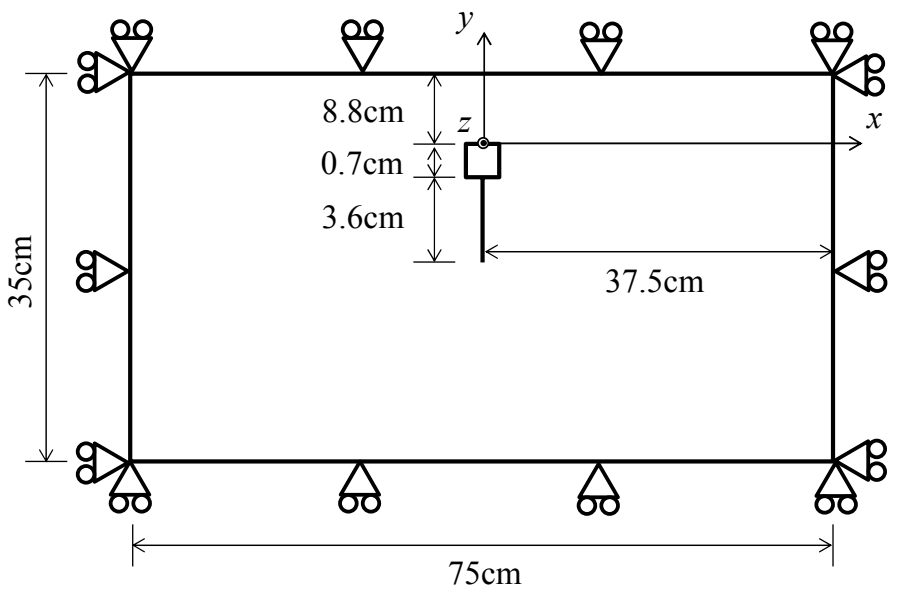

Figure 2: Schematic of the problem setup.
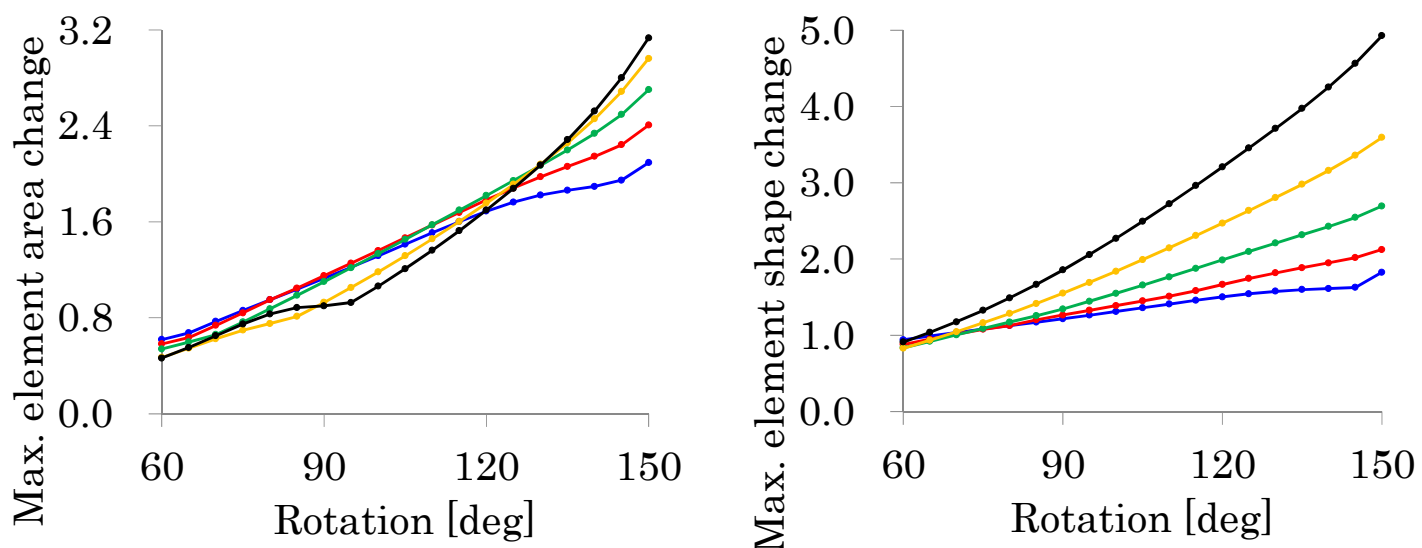

$$
\begin{aligned}
& \rightarrow(0.3,0.7) \rightarrow(0.4,0.6) \rightarrow(0.5,0.5) \\
& \rightarrow(0.7,0.3) \rightarrow(1.0,0.0)
\end{aligned}
$$$$
\rightarrow(0.3,0.7) \rightarrow(0.4,0.6) \rightarrow(0.5,0.5)
$$$$
\rightarrow(0.7,0.3) \rightarrow(1.0,0.0)
$$

Figure 3: Maximum element area change Figure 4: Maximum element shape change plotted against structure rotations ranging plotted against structure rotations ranging from $60^{\circ}$ to $150^{\circ}$ for different $\left(\chi_{1}, \chi_{2}\right)$ values. from $60^{\circ}$ to $150^{\circ}$ for different $\left(\chi_{1}, \chi_{2}\right)$ values.

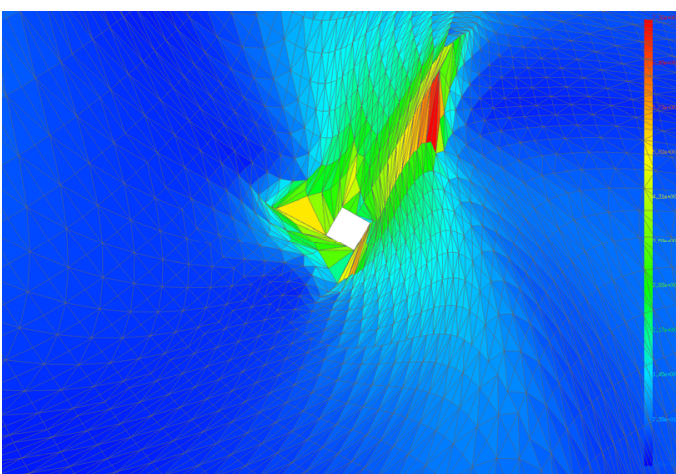

Figure 5: Equivalent shear strain near the structure for the Jacobian-based stiffening.

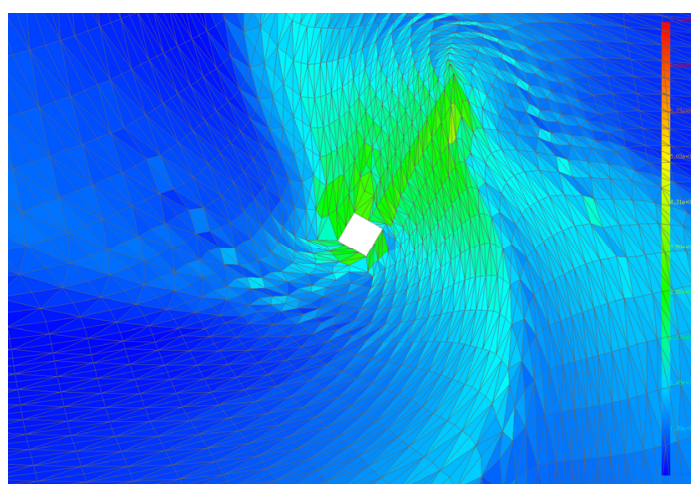

Figure 6: Equivalent shear strain near the structure for $\left(\chi_{1}, \chi_{2}\right)=(0.3,0.7)$. 


\section{Journal of Advanced Simulation in Science and Engineering}

\subsection{Three-dimensional rotation}

A rectangular domain including a rigid body consisting of a rectangular cylinder and a fin was considered, as shown in Fig. 7. This problem is the extension of the previous problem shown in Fig. 2 along the direction of the $z$-axis. Therefore, the $x y$-plane view of the mesh decomposition shown in Fig. 7 (c) is equivalent to that for the previous problem. This domain was descritized using linearly interpolated tetrahedral elements with the number of nodes and elements 46911 and 254352 , respectively. A rotational displacement $\theta_{z}$ about the $z$-axis, which corresponds to the feathering motion, was applied to the rigid body incrementally. The amplitude of $\theta_{z}$ changes from $10^{\circ}$ to $110^{\circ}$. In addition, a rotational displacement $\theta_{y}$ about the $y$-axis, which corresponds to the flapping motion, was applied to the rigid body incrementally [4]. The amplitude of $\theta_{y}$ is set as $60^{\circ} \cdot \chi_{3}$ for each $\left(\chi_{1}, \chi_{2}\right)$ values was obtained as the value that minimizes the mesh quality measure of (3).

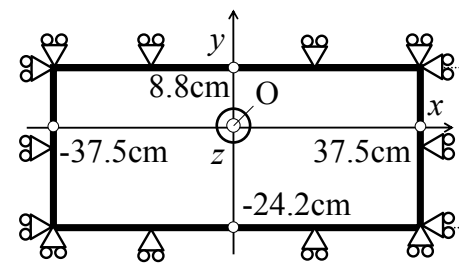

(a) The fluid domain

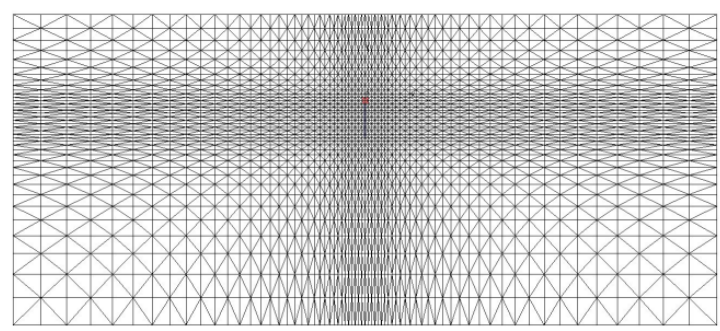

(c) The $x y$-plane view of the mesh decomposition

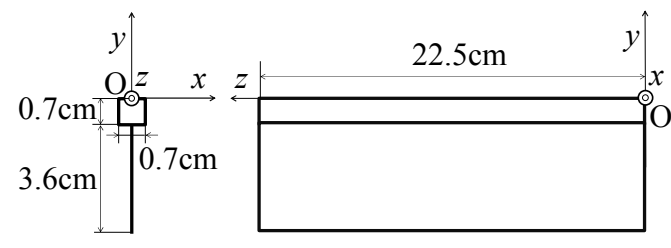

(b) The wing

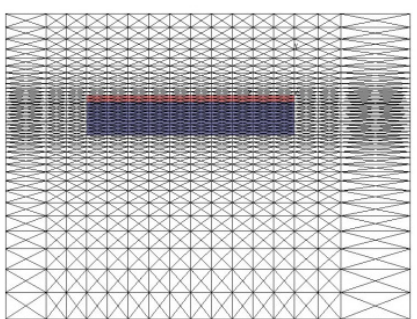

(d) The $y z$-plane view of the mesh decomposition

Figure 7: Schematic of the problem setup.

Figures 8 and 9 show the changes of the mesh quality measures plotted against the rotation $\theta_{z}$. As shown in these figures, the stiffening coefficient with $\left(\chi_{1}, \chi_{2}\right)=(0.7,0.3)$ showed the best performance to maintain the element area and shape under large rotations. Note that the stiffening coefficient with $\left(\chi_{1}, \chi_{2}\right)=(0.4,0.6)$ showed the minimum value of the maximum element shape change under large rotations, but it failed for $\theta_{z}$ after $110^{\circ}$. From this observation, the proposed scenario with $\chi_{1}>\chi_{2}$ shows better performance than the previous stiffening in terms of maintaining the mesh quality under the large feathering and flapping motion. In the case of the feathering motion, the mesh was twisted around the structure. Similar to the previous section, this type of motion induced a large shear deformation in the vicinity of the structure. Therefore, the proposed scenario can improve the mesh quality because of the weighted evaluation for the area and the aspect ratio of each element according to the magnitude of the shear deformation. 


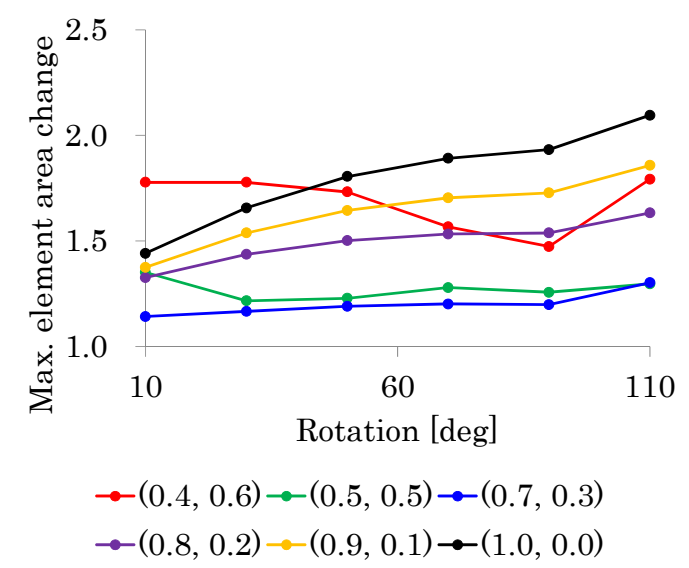

Figure 8: Maximum element area change plotted against structure rotations ranging from $10^{\circ}$ to $110^{\circ}$ for different $\left(\chi_{1}, \chi_{2}\right)$ values.

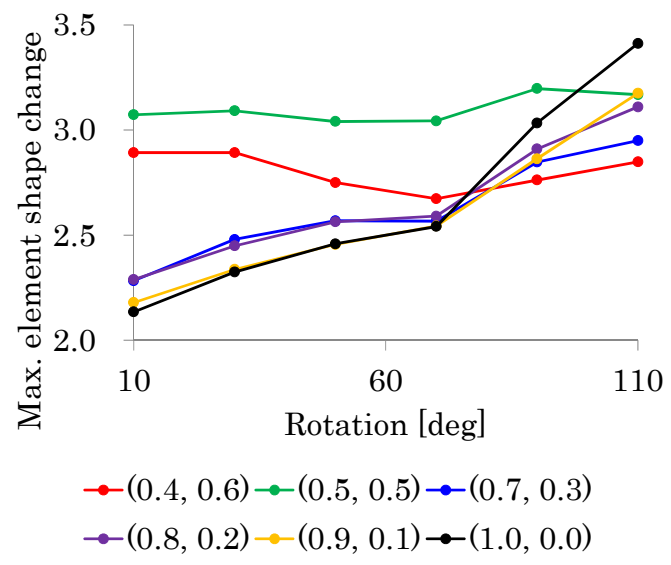

Figure 9: Maximum element shape change plotted against structure rotations ranging from $10^{\circ}$ to $110^{\circ}$ for different $\left(\chi_{1}, \chi_{2}\right)$ values.

\section{Concluding remarks}

The proposed scenario of the selective mesh stiffening in the pseudoelastic mesh-moving is based on two element quality parameters, the element area and shape, which are in a tradeoff relationship. Importantly, this scenario includes the previous stiffening as a specific case. To demonstrate the performance of the proposed scenario, it was applied to the mesh-moving of a rectangular domain including a structure consisting of a square and a fin undergoing large rotations. This type of motion induces a large shear deformation in the vicinity of the structure. Therefore, the proposed scenario shows better performance than the previous stiffening in terms of maintaining the mesh quality, since it uses the weighted evaluation for the area and the aspect ratio of each element according to the magnitude of the shear deformation.

\section{Acknowledgement}

This work was supported by JSPS KAKENHI Grant Number $17 \mathrm{H} 02830$.

\section{References}

[1] Stein, L. Tezduyar, T. Benney, R: Automatic mesh update with the solid-extension mesh moving technique, Computer Methods in Applied Mechanics and Engineering, 193 (2004), 2019-2032.

[2] Yamada, T. Yamamoto, Y. Hong, G. Yoshimura, S: A mesh moving technique with minimum-height-based stiffening for fluid-structure interaction analysis, Mechanical Engineering Letters, 3 (2017), 16-00657 (8 pages).

[3] Ishihara, D. Horie, T. Denda, M: A two-dimensional computational study on the flu- 


\section{Journal of Advanced Simulation in Science and Engineering}

id-structure interaction cause of wing pitch changes in dipteran flapping flight, The Journal of Experimental Biology, 212 (2009), 1-10.

[4] Ishihara, D. Horie, T. Niho, T: An experimental and three-dimensional computational study on the aerodynamic contribution to the passive pitching motion of flapping wings in hovering flies, Bioinspiration \& Biomimetics, 9 (2014), 046009 (23pp). 